\title{
Status of the TRIUMF annular chamber for the tracking and identification of charged particles (TACTIC)
}

\author{
G. Ruprecht ${ }^{1, a}$, D. Gigliotti ${ }^{1}$, P. Amaudruz ${ }^{1}$, L. Buchmann ${ }^{1}$, S.P. Fox ${ }^{2}$, B.R. Fulton ${ }^{2}$, T. Kirchner ${ }^{1}$, A.M. Laird ${ }^{2}$, \\ P.D. Mumby-Croft ${ }^{2}$, R. Openshaw ${ }^{1}$, M.M. Pavan ${ }^{1}$, J. Pearson ${ }^{1}$, G. Sheffer ${ }^{1}$, and P. Walden ${ }^{1}$ \\ 1 TRIUMF, 4004 Wesbrook Mall, Vancouver, BC, V6T 2A3, Canada \\ 2 University of York, Heslington, York, YO10 5DD, UK
}

Received: 1 July 2005 /

Published online: 15 March 2006 - (C) Società Italiana di Fisica / Springer-Verlag 2006

\begin{abstract}
TACTIC (TACTIC web site: http://tactic.triumf.ca) is a new detector for low-energy nuclear reactions currently under development at TRIUMF. The cylindrical ionization chamber allows threedimensional reconstruction of particle tracks by means of a two-dimensional anode array combined with a TOF measurement of the drift electrons. In addition, the integrated charge for each pulse provides information about the energy loss of the particle and therefore allows a better identification of the nuclear species producing the track. The geometry of TACTIC covers a large angular range permitting the measurement of differential cross-sections over a large solid angle. It will be ideal for investigations of nuclear processes pertinent to the field of nuclear astrophysics.
\end{abstract}

PACS. 25.55.-e ${ }^{3} \mathrm{H}-,{ }^{3} \mathrm{He}$-, and ${ }^{4} \mathrm{He}-\mathrm{induced}$ reactions - 29.40.Cs Gas-filled counters: ionization chambers, proportional, and avalanche counters - 29.40.Gx Tracking and position-sensitive detectors

\section{Introduction}

With the advent of radioactive ion beam (RIB) facilities many reactions have to be measured in inverse kinematics. For capture reactions, the cone of the ejected heavy ions is usually sufficiently small so that a recoil separator can be used for detecting $100 \%$ of the ions. For reactions with two or more heavy ejectiles, the cone is larger and a detector array like TUDA [1] is the better option. However, a fraction of the angular range is lost for small angles in order to let the beam through, and for larger angles where the ejectiles do not reach the detector. When using gas targets, if the energy of the ejectiles is low they cannot penetrate the gas and/or the exit window and also lose energy in the dead layer of the detector.

Another approach to cover a large forward angular range is to use an ionization chamber. The problem here is that the target and detection region are not separated, resulting in a large background and poor statistics. High segmentation is needed in order to collect as many track points as possible and this would require several amplifying gas cells as well as amplification and digitalization electronics.

Our approach is to employ Gas Electron Multiplier (GEM) [2,3] foils for the first stage of amplification inside the chamber. This considerably reduces the complexity

\footnotetext{
a e-mail: ruprecht@triumf.ca
}

of a cylindrical chamber design which in turn makes a separation of target and detection region more feasible.

\section{The TACTIC chamber}

The TRIUMF Annular Chamber for the Tracking and Identification of Charged Particles (TACTIC) is a combined cylindrical ionization (IC)/time projection chamber (TPC) detector where the gas target along the central axis can be "windowless" to ejectiles (i.e. the target and detector gases are the same), or "windowed" (i.e. a thin window separates disparate target and detector gases). In either case field-defining cathode wires delimit the target region. Using this method, the beginning part of the track within the target region cannot be "seen" in the drift region, but the vertex point can still be inferred by extrapolating the reconstructed TPC anode hits. The total energy deposited by the stopping ejectiles is also measured by the accumulated charge on the anodes. Furthermore, using the vertex reconstruction, the energy loss of the beam in the target allows the simultaneous measurement of cross-sections and angular distributions over a range of energies.

The GEM foil $[2,3]$ acts as a preamplifier inside the chamber wall providing low-noise signals requiring only one further stage of amplification. The signals are digitized using a multi-channel VME-based flash ADC board, thus minimizing the amount of electronics required. 


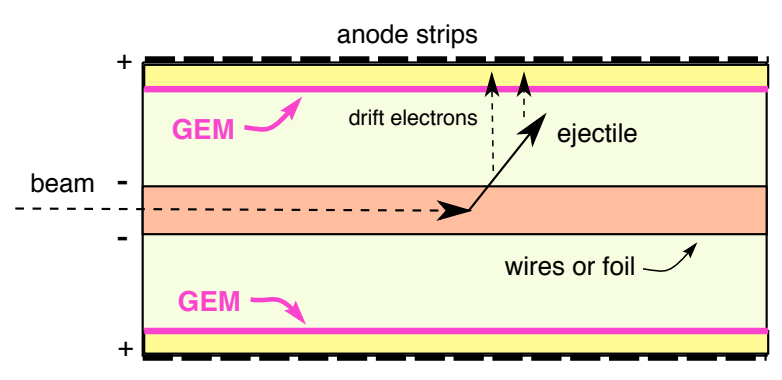

Fig. 1. Schematic side view of the proposed TACTIC detector.

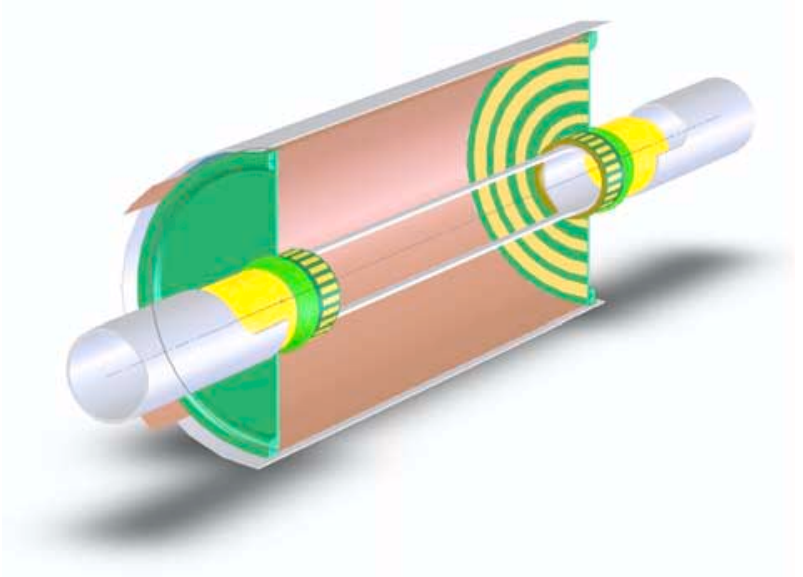

Fig. 2. Three-dimensional cut-away view of the proposed TACTIC detector.

A schematic view of the chamber is shown in fig. 1. Target and drift region are separated by wires or a foil (depending on the experiment) acting as the cathode for the drift electrons. The ejectiles move into the drift region where they produce electrons along the track which drift slowly (compared to the flight time of the ejectile) towards the GEM surrounding the entire drift cylinder. These primary electrons create an electron avalanche in the high electric field inside the GEM, producing a signal gain of 10-100. The avalanche electrons are collected by the nearby anode pads on the chamber wall. Like a TPC, the time differences of the signals between the pads gives information about the trajectory, whereas like an IC the collected charge is a measure of the ejectile energy loss. The trajectory together with the released charge along the track allows a unique identification of the nuclei ejected, which is essential for experiments involving ejectiles with similar energy but different charges or masses.

In the three-dimensional view, fig. 2, the support structure for the cathode wires (or target foil) can be seen. There are also biased rings at the end caps to straighten the drift field (see also sect. 5). The anode pads (not shown) are sub-divided azimuthally. This is necessary for high counting rates caused either by elastically scattered nuclei or decaying beam particles but may be also required by special experiments like reactions with polarized beam or with several heavy-ion ejectiles. The pads are etched onto flexible $\mathrm{PCB}$ which also holds the electrical connec-

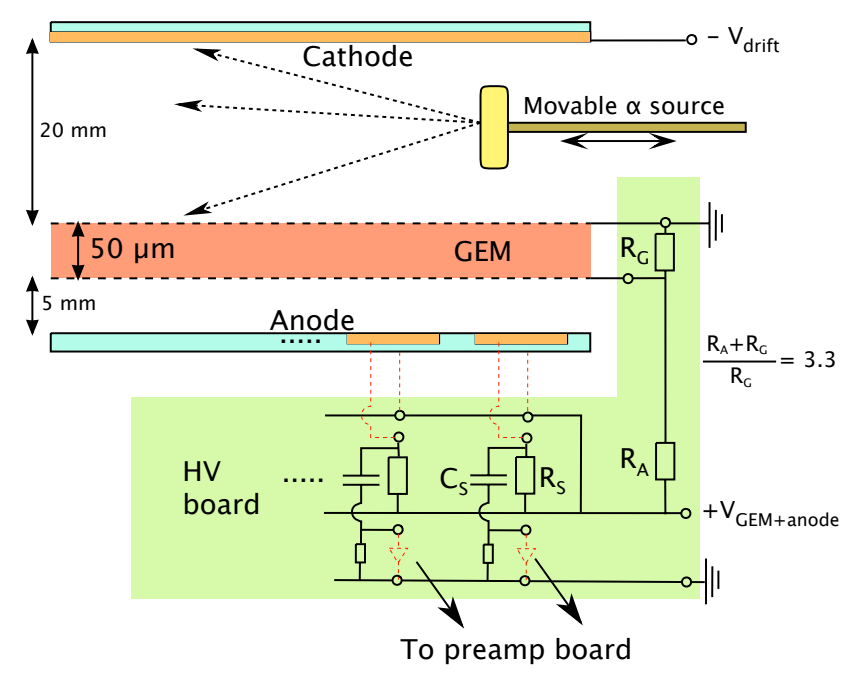

Fig. 3. Schematic view of the planar TACTIC test chamber investigating the properties of the GEM and testing particle tracking.

tors. The chamber will be constructed as two half cylinders allowing the target tube, including the separation wires or foil, to be removed or replaced. The chamber is placed in a detector-gas filled outer box that connects to the beam tube.

\section{The TACTIC test chamber.}

For the proposed first experiment, ${ }^{8} \operatorname{Li}(\alpha, n){ }^{11} \mathrm{~B}$, the energy of the recoils for the interesting part of the excitation curve is too small to penetrate a foil separating the detection region from the drift region, so a set of wires will replace the foil. This requires the target gas (primary helium) to be working as detection gas as well. In order to investigate the dependence of the GEM gain on the helium mixture and gas pressure, and to determine the local resolution of the particle track points, tests have been performed on a planar test chamber (see fig. 3). A movable $5486 \mathrm{keV}$ alpha particle source was mounted perpendicular to the strips. At lower pressures a $16 \mu \mathrm{m}$ Mylar foil was mounted in front of the source to reduce the alpha particle range.

The test chamber has a drift volume of about $20 \times 20 \times$ $2 \mathrm{~cm}^{3}$ and an active GEM area of $8 \times 8 \mathrm{~cm}^{2}$, covering 16 active anode strips, each $5 \mathrm{~mm}$ in width. The anodes are under positive high voltage and decoupled by capacitors. The signals are amplified by a single 16-channel preamplifier board. Analogue electronics and CAMAC ADCs and TDCs have been used to process the signals. We measured the change of the GEM gain for different mixtures of $\mathrm{Ar} / \mathrm{CO}_{2}$ as well as $\mathrm{He} / \mathrm{CO}_{2}$. The $\mathrm{CO}_{2}$ quenching gas fraction was adjusted by a gas handling system (GHS). The highest amplification was achieved with a 90\%/10\% $\mathrm{He} / \mathrm{CO}_{2}$ ratio. The $\mathrm{He} / \mathrm{CO}_{2}$ mixture was comparatively better than an $\mathrm{Ar} / \mathrm{CO}_{2}$ mixture which is often used as a detector gas (see fig. 4). 


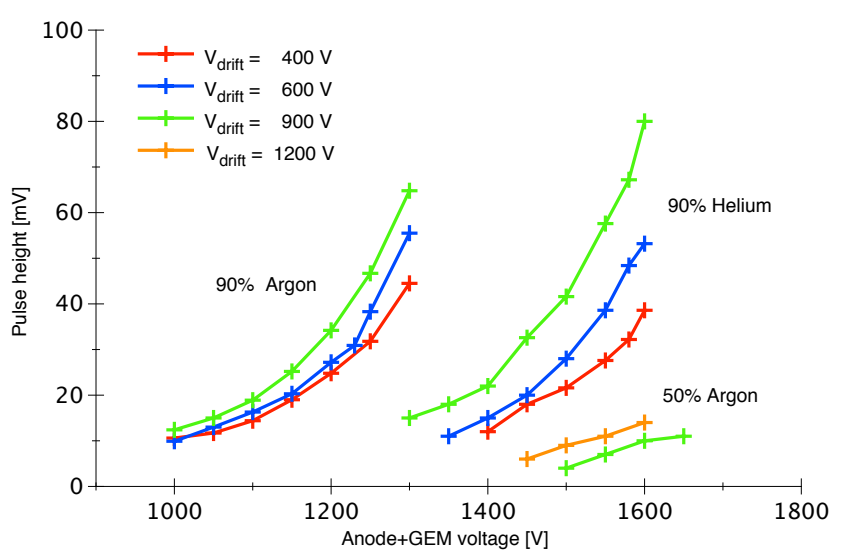

Fig. 4. Pulse heights vs. GEM+Anode voltage (the GEM voltage is a factor of 3.3 smaller).

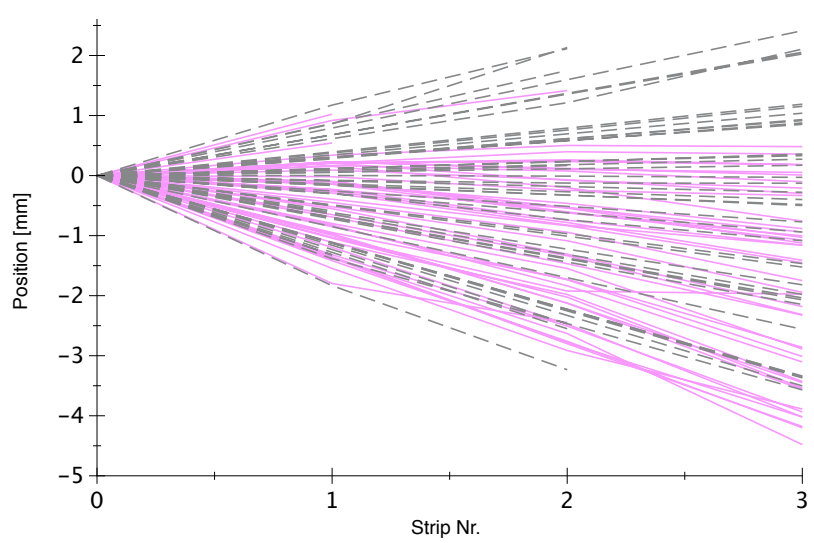

Fig. 5. (Colour on-line) Electron drift times with respect to the first strip, converted to distances assuming a drift velocity of $12 \mathrm{~mm} / \mu \mathrm{s}$. The electrons are released along the tracks of the $\alpha$ 's. The dashed lines stem from a GEANT4 simulation, the solid purple lines from the measurement.

By measuring the time differences between the signals on 4 consecutive strips we have been able to gain a projected image of the particle tracks, as can be seen in fig. 5 . One observes good agreement between measurement and simulation. The source was mounted $5 \mathrm{~cm}$ before the first strip. The first strip signal was used as the trigger, so all track times are with respect to the first strip.

In a second phase, the gas pressure was varied down to 100 mbar while keeping the $\mathrm{He} / \mathrm{CO}_{2}$ mixture at a constant ratio (below 100 mbar the oxygen contamination becomes too high and quenches the signals) and the flow rate at $200 \mathrm{~cm}^{3} / \mathrm{min}$. This enabled the dependence of the GEM gain on pressure and applied voltage to be determined. The source was not collimated, therefore particles were emitted in all directions producing a broad energy spectrum at each anode. The relative gain was found by comparing the measured spectra with the results of a GEANT4 [4] simulation. While the shape of the gain vs. GEM voltage curves remains nearly the same, the gain at a constant GEM voltage scales approximately with the square root of pressure, as does the breakdown voltage (see

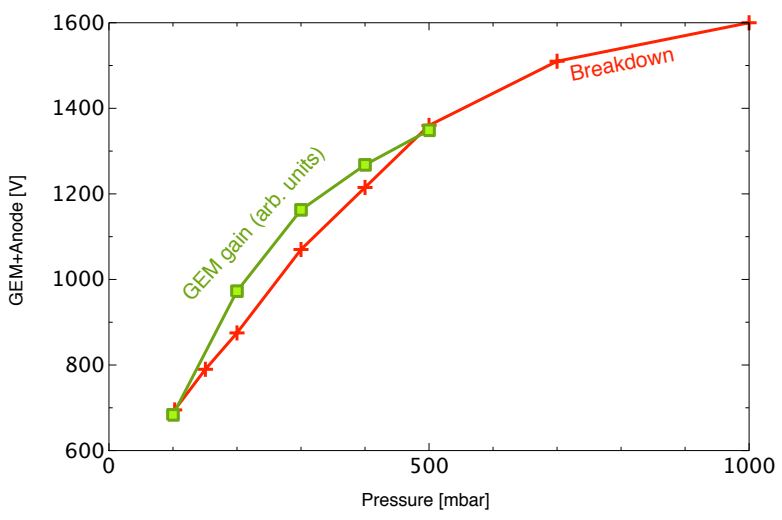

Fig. 6. The GEM trip voltage, and the relative gain at a constant GEM voltage, are shown as a function of gas pressure. Both nearly scale with the square root of pressure.

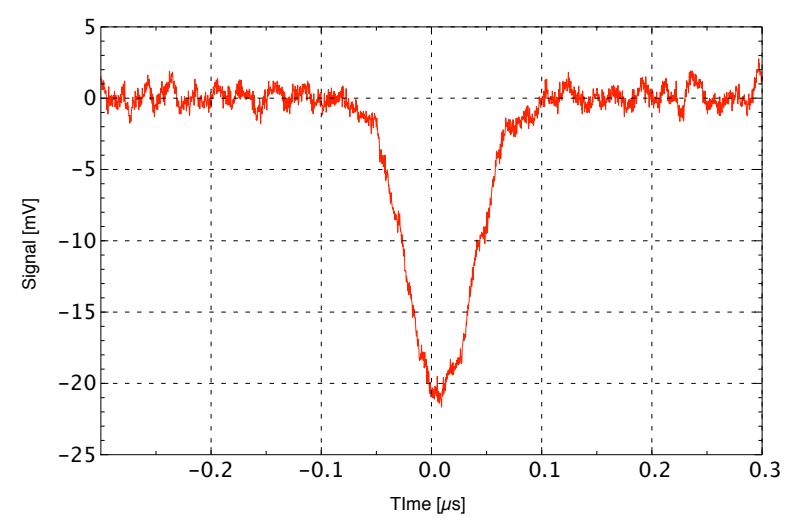

Fig. 7. Amplified signal of $\approx 5 \mathrm{MeV} \alpha$ particles at 500 mbar.

fig. 6). This is a useful result for the design of the cylindrical chamber. A typical signal is shown in fig. 7. Despite noise in the laboratory environment and the very weak initial ionization, the signals look very clear and promising.

\section{GEANT4 simulation}

A change of the gas pressure affects the initial ionization per anode strip, the track length and straggling of the ejectiles, the energy loss and straggling of the projectiles in the target region, as well as the gain of the GEM. To study the mutual dependence of all these parameters a MonteCarlo simulation of all relevant processes taking part in the chamber was performed using the GEANT4 framework [4]. The most important questions to be answered from the simulation are

- How accurately can the reaction vertex point be reconstructed from the anode signals? This is important because the interaction energy must be well known for measurements below the Coulomb threshold.

- What is the angular resolution?

- What is the energy resolution?

- When the emission of low-energetic gammas is expected, what attenuation can be expected at the gamma detectors placed outside the chamber? 


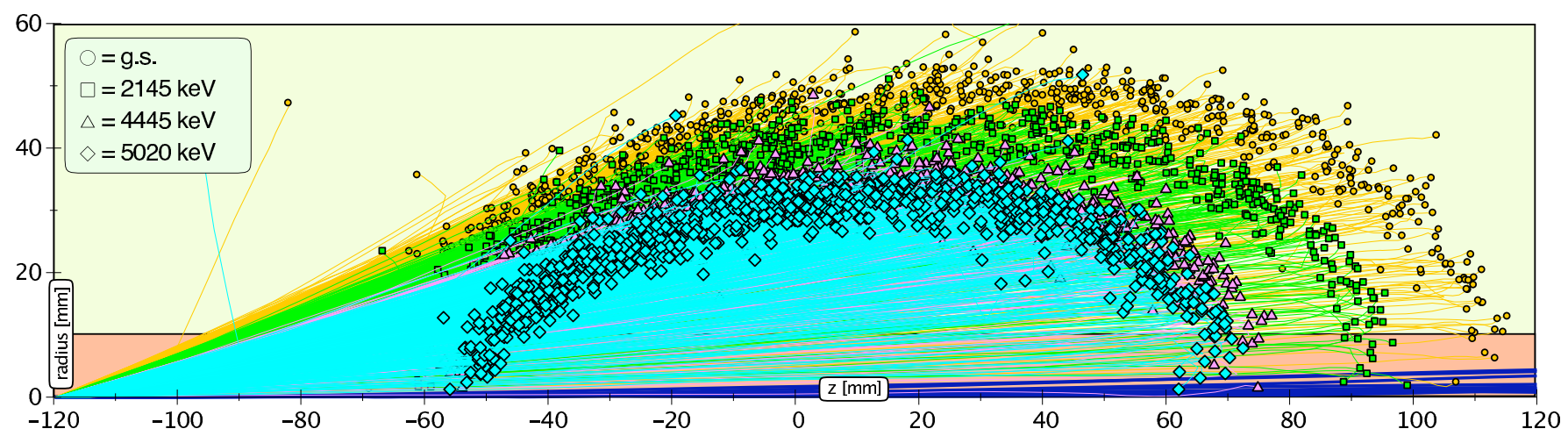

Fig. 8. (Colour on-line) Trajectories and end-point distribution calculated with GEANT4 of the ${ }^{11} \mathrm{~B}$ ejectiles for the ground state and the first three excited states. The simulated detector is filled with a $90 / 10 \mathrm{He} / \mathrm{CO}_{2}$ gas mixture at room temperature and a pressure of 250 mbar. The target region ranges from $r=0-10 \mathrm{~mm}$ (red background). The $9 \mathrm{MeV}{ }^{8} \mathrm{Li}$ beam comes from the left-hand side and hits the target at $z=-120 \mathrm{~mm}$ (thick blue lines). Only the ejectiles that stem from $8.9-9.0 \mathrm{MeV}$ beam energies are shown.

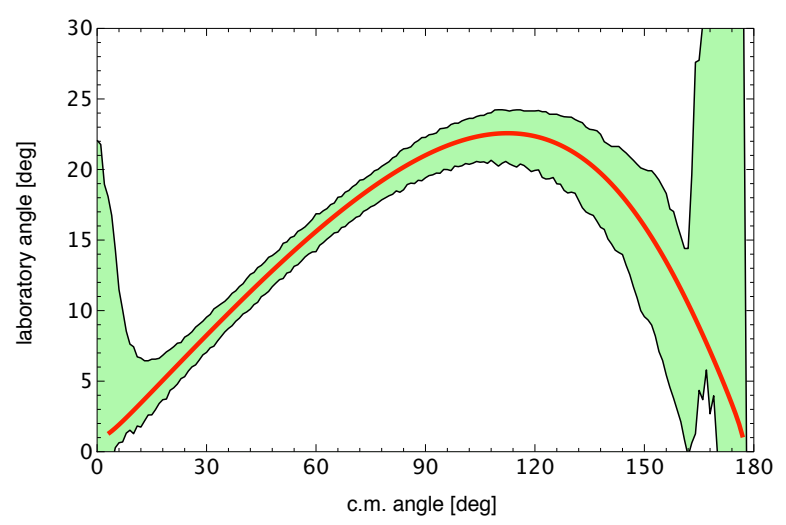

Fig. 9. Uncertainty ( $\pm \sigma$ range calculated for 10000 events) of the ${ }^{11} \mathrm{~B}$ laboratory angles, reconstructed using 48 anode rings, each $5 \mathrm{~mm}$ in width, emitted at different c.m. angles as expected from the GEANT4 simulation for the ${ }^{11} \mathrm{~B}$ ground state. Overlayed is the theoretical curve.

GEANT4 is tailored for high-energy physics, and support for low-energy nuclear reactions is limited. There are currently problems with the energy loss of ions at low energies and more work in this area is needed, but some preliminary results are presented here.

The geometry is defined by the maximum of the $z$ - and $r$-projected ejectile range, where $z$ is the coordinate along beam axis and $r$ the radial distance. The simulated tracks of the ejectiles are shown in fig. 8 for $E_{\text {beam }}=9 \mathrm{MeV}$ and 4 different ${ }^{11} \mathrm{~B}$ states ${ }^{1}$. Regarding the endpoints, for small laboratory angles and high energies the ${ }^{11} \mathrm{~B}$ levels can be better resolved than for lower energies corresponding to a backwards emission in the centre-of-mass (c.m.) frame, or larger angles. However, combined with the measured ejectile energies, there is some improvement possible.

The angular resolution using 48 anode rings can be seen in fig. 9. The diagram shows the standard deviation around the true angle in the c.m. system. For a measured

\footnotetext{
${ }^{1}$ For the energies involved here the stopping powers and ranges are in agreement with the results from SRIM [5].
}

laboratory angle of $20^{\circ}$ there is a large uncertainty for the c.m. angle (between $70^{\circ}$ and $150^{\circ}$ ). This calculation is based on a simple linear least squares fit of the radial distances expected to be measured with the anodes (the error of the drift time is not currently included in the simulation). The uncertainty comes from the $z$-resolution as well as from the beam and ejectile straggling. With a more sophisticated analysis (e.g. giving smaller radii more weight) an improvement is possible. Again, no energy signals have been taken into account which can further refine the analysis.

The simulation is a crucial contribution for the detector design as well as for later experimental analysis. Further refinements are required to be able to obtain reliable statements about the vertex reconstruction and energy resolution. The estimates presented here have been done without taking the drift time uncertainties into account. The latter can be calculated using GARFIELD [6]. Combining GEANT4 with GARFIELD, a simulation of the anode signal shape can be achieved providing a further piece of information from the particles.

We have implemented the software for low-energy reactions $(\mathrm{C}++$ objects $)$ in a generic manner that can be used for other GEANT4 applications as well. As detectors for nuclear reactions become more complex, this will be a helpful contribution to the GEANT4 repository that other nuclear physics groups could use for detector simulation or analysis.

\section{Drift field and beam induced electrons}

Electrons induced directly by the beam could potentially drift from the target region to the detection region where they produce unwanted signals. To avoid this, a second cage of wires held at a slightly more positive voltage will be inserted a few mm inside the cathode wire cage, fully encompassing the expected incident beam spread. A GARFIELD [6] calculation has shown that electrons released in the target region will be collected at the inner cage and not drift to the detection region (see fig. 10). 


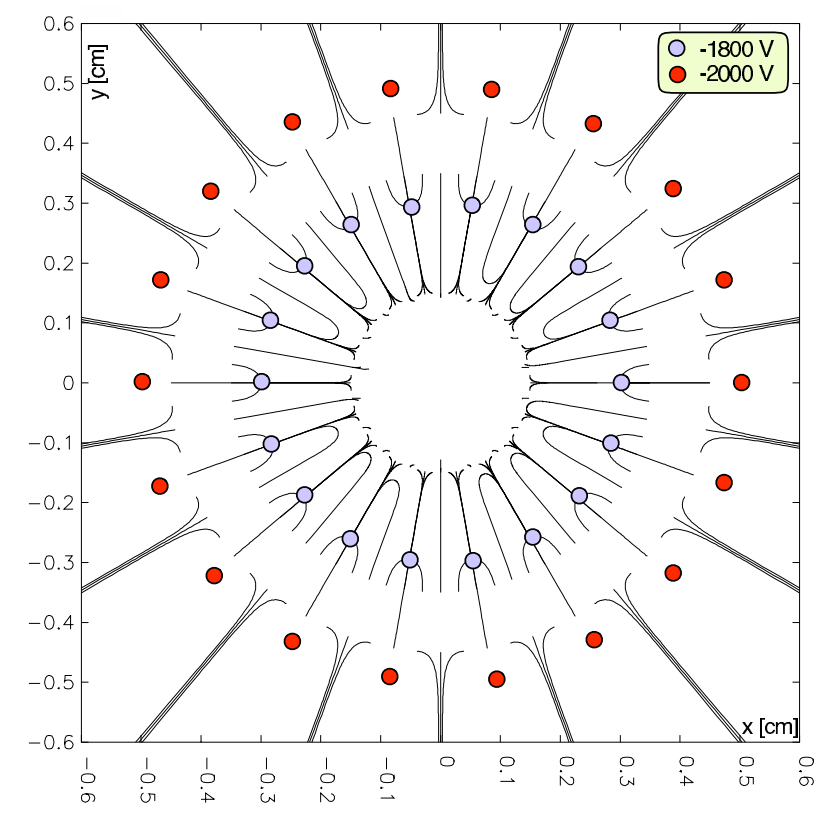

Fig. 10. GARFIELD simulation of the electron drift in the target region. The circles mark the wires, and the thin black lines the paths of the electrons released at different positions. Only electrons close to the cathode wire cage reach escape the target region and enter the detection volume.

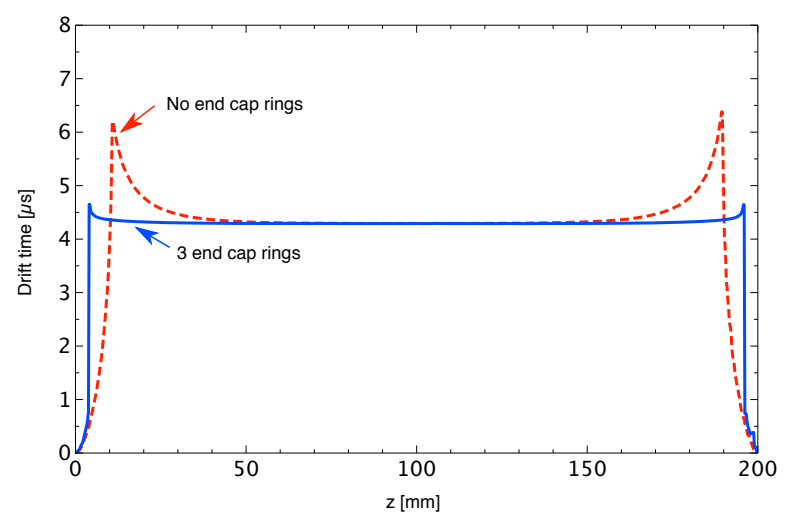

Fig. 11. Drift time of electrons released at $-500 \mathrm{~V}$ biased cathode wires to the GEM vs. the $z$-axis, with and without voltage-shaping rings at the end caps at $10 \mathrm{~mm}$ radius. The radii of the cathode cage and of the GEM cylinder are $10 \mathrm{~mm}$ and $50 \mathrm{~mm}$, respectively.

An accurate measurement of the drift time demands a uniform electric field within TACTIC. Since the enclosure outside the end caps is electrically grounded, the drift field is distorted close to the end caps. Therefore, the field near the endcaps must be shaped by appropriately biased rings (see fig. 2). Figure 11 shows the field uniformity improvement by using three rings. The electric field has been calculated using FEMLAB [7] and the total drift time was obtained by integrating along the field lines, assuming a linear approximation for the drift velocity given in [8] for a 90/10 mixture of $\mathrm{Ar} / \mathrm{CO}_{2}$.

\section{Data acquisition}

We will use flash ADCs for the data acquisition - a new technique that is becoming more popular for nuclear physics applications. The entire anode signal is sampled and can be stored for later analysis. For the TACTIC prototype we will use a 48-channel VME board with a sampling rate of $40-70 \mathrm{MHz}$ and a resolution of 10 bits per sample. However, due to the large data rate it is desirable to extract time and charge information immediately proceeding the digitalization. The firmware running on the VME board offers a large number of configuration possibilities based on custom analysis algorithms. This feature is required because the pulse shapes differ depending on the direction of the recoil.

The raw charge and time data provided by the flash ADC board will be stored and pre-analysed using TRIUMF's MIDAS [9] system. DAQ systems for nuclear physics usually lack the ability to display particle tracks, so a more sophisticated analysis system, ROOT [10], will be used. There also exists a new MIDAS/ROOT interface, ROME [11], which is still under development at PSI, Switzerland. We are currently testing the suitability of these tools for this kind of particle tracking experiment.

\section{Applications}

The first experiment planned to be measured with TACTIC is the ${ }^{8} \operatorname{Li}(\alpha, n)^{11} \mathrm{~B}$ reaction which plays a role in $\mathrm{r}$ process nucleosynthesis [12]. Including light elements in a scenario of neutrino-driven wind reactions with light nuclei can change the synthesis of heavier elements by an order of magnitude. One important reaction chain is $\alpha(\mathrm{t}, \gamma)^{7} \mathrm{Li}(\mathrm{n}, \gamma)^{8} \mathrm{Li}(\alpha, \mathrm{n})^{11} \mathrm{~B}$. The total cross-section at $9 \mathrm{MeV}^{8} \mathrm{Li}$ impact energy is about $400 \mathrm{mb}$ but only a fraction $(\approx 20 \%)$ goes into the ${ }^{11} \mathrm{~B}$ ground state, while there are up to 8 excited states involved.

In a recently published measurement [13], an ion chamber filled with a $90 / 10 \mathrm{He} / \mathrm{CO}_{2}$ gas mixture and $2 \times 64$ flash ADCs for the readout enabled a three-dimensional reconstruction of the tracks. The resulting energy spectra allowed a rough separation of the ${ }^{11} \mathrm{~B}$ levels. In contrast, TACTIC uses a GEM which, in principle, allows for much better local resolution and therefore better tracking. The layout of the TACTIC prototype will be designed to enclose the ${ }^{11} \mathrm{~B}$ tracks for ${ }^{8} \mathrm{Li}$ impact energies between 1.2 and $9.0 \mathrm{MeV}$.

Another experiment is the measurement of the ${ }^{7} \mathrm{Be}+\mathrm{p}$ elastic scattering cross-section at low energies. The angular distributions give information on the phase shifts for different angular momentum and spin combinations. There are 16 phase shifts involved if only $s, p$, and $d$-waves are taken into account, therefore a high angular resolution is required for this experiment. The contradicting results when compared with the mirror reaction ${ }^{7} \mathrm{Li}+\mathrm{n}[14$, $15,16]$ could be resolved by an accurate angular distribution measurement using TACTIC. A better understanding of the resonance structure will help in extrapolating the ${ }^{7} \mathrm{Be}(\mathrm{p}, \gamma){ }^{8} \mathrm{~B}$ cross-section to low energies - an important 
reaction for the high-energy neutrino production in the Sun.

Another interesting application is the measurement of nuclear reactions with three or more heavy-ion ejectiles. The more complex phase space requires a large angular range with a good energy resolution to be measured for two or more ejectiles in coincidence. This is hard to achieve with passive detectors like silicon counters, while in a TACTIC-like detector a high fraction of the ejectiles can be tracked simultaneously.

\section{Conclusions}

A cylindrical ionization chamber layout in combination with GEM foils for time projection chamber-like tracking is a promising detector configuration for low-energy nuclear reactions, in particular for measurements in inverse kinematics. The GEM is easy to handle and there are fewer restrictions as opposed to gas cell pads. Moreover, it works very well with a $\mathrm{He} / \mathrm{CO}_{2}$ gas mixture which opens the possibility of ${ }^{4} \mathrm{He}$ and ${ }^{3} \mathrm{He}$ induced $\mathrm{RIB}$ reactions at low beam energies.

The suppression of the ionization electrons created by the beam reduces the event rate by orders of magnitude and makes TACTIC suitable for high-current RIB facilities like ISAC/TRIUMF. The first measurement - the reaction ${ }^{8} \mathrm{Li}(\alpha, \mathrm{n})^{11} \mathrm{~B}$ - will show the actual resolving power of the TACTIC prototype and the maximum counting rate that can be achieved.
The capability of simultaneous particle tracking qualifies TACTIC for reactions with three or more heavy-ion ejectiles. For this, however, a higher azimuthal segmentation of the anodes is required but it remains an interesting application for the future.

\section{References}

1. TUDA web site: http://tuda.triumf .ca.

2. F.Sauli, A. Sharma, Annu. Rev. Nucl. Part. Sci. 49, 341 (1999).

3. A. Sharma, F. Pauli, Nucl. Instrum. Methods A 350, 470 (1994).

4. GEANT4 web site: http://cern.ch/geant4.

5. SRIM web site: http://www.srim.org.

6. GARFIELD web site: http://garfield.web.cern.ch.

7. FEMLAB web site: http://www.comsol.com/products/ femlab.

8. A. Peisert, F. Sauli, Drift and Diffusion of Electrons in Gases, CERN, 1984.

9. MIDAS web site: http://midas.triumf.ca.

10. ROOT web site: http://root.cern.ch.

11. ROME web site: http://midas.psi.ch/rome.

12. M. Terasawa, K. Sumiyoshi, T. Kajino, J. Mathews, I. Tanihata, Astrophys. J. 562, 470 (2001).

13. Hashimoto et al., Nuc. Phys. A 764, 330c (2004).

14. C. Angulo et al., Nucl. Phys. A 716, 211 (2003).

15. G.V. Rogachev et al., Phys. Rev. C 64, 061601 (2001).

16. F. Barker, A.M. Mukhamezhanov, Nucl. Phys. A 673, 526 (2000). 